\title{
909.
}

\section{ON A PARTICULAR CASE OF KUMMER'S DIFFERENTIAL EQUATION OF THE THIRD ORDER.}

[From the Messenger of Mathematics, vol. xx. (1891), pp. 75-79.]

THE general form of equation in question is

$$
\frac{x^{\prime \prime \prime}}{x^{\prime}}-\frac{3}{2}\left(\frac{x^{\prime \prime}}{x^{\prime}}\right)^{2}+x^{\prime 2}\left\{\frac{A}{(x-1)^{2}}+\frac{B}{x(x-1)}+\frac{C}{x^{2}}\right\}-\left\{\frac{A^{\prime}}{(t-1)^{2}}+\frac{B^{\prime}}{t(t-1)}+\frac{C^{\prime}}{t^{2}}\right\}=0,
$$

here $x$ is a function of $t$; and $A, B, C, A^{\prime}, B^{\prime}, C^{\prime}$ are numerical constants. For various given values of $A, B, C$, and values determined thereby of $A^{\prime}, B^{\prime}, C^{\prime}$, the equation admits of a solution in the form $x=$ rational function of $t$; the theory in reference to the cases considered by Schwarz is considered in my paper "On the Schwarzian Derivative and the Polyhedral Functions," Camb. Phil. Trans., t. xIII. (1883), pp. 5-68, [744]. But the theory is considered in a more general and exhaustive manner in Goursat's memoir, "Recherches sur l'équation de Kummer," Acta Soc. Sci. Fennica, t. xv. (1888), pp. 47-127. I consider here one of the solutions given by him, viz. writing

$$
\begin{array}{ll}
P=4 t-5 \quad, \quad X=t^{2} P^{3} \\
Q=5 t-4 \quad, \quad Y=Q^{3} \\
R=8 t^{2}-11 t+8, \quad Z=-(t-1)^{2} R^{2}
\end{array}
$$

so that, identically, $X+Y+Z=0$; then the solution is expressed by either of the equivalent equations

$$
\begin{aligned}
& x=-\frac{X}{Z}=\frac{t^{2} P^{3}}{(t-1)^{2} R^{2}}, \\
& x-1=\frac{Y}{Z}=-\frac{Q^{3}}{(t-1)^{2} R^{2}}
\end{aligned}
$$


The values of the constants to which this solution belongs are

$$
A=\frac{4}{9}, \quad B=-\frac{37}{32}, \quad C=\frac{4}{9} ; A^{\prime}=\frac{3}{8}, \quad B^{\prime}=\frac{131}{144}, C^{\prime}=\frac{5}{18} .
$$

But instead of assuming these values in the first instance, I leave the values indeterminate; and starting from the foregoing expression for $x$, I substitute this in

$$
\Omega=\frac{x^{\prime \prime \prime}}{x^{\prime}}-\frac{3}{2}\left(\frac{x^{\prime \prime}}{x^{\prime}}\right)^{2}+x^{\prime 2}\left\{\frac{A}{(x-1)^{2}}+\frac{B}{x(x-1)}+\frac{C}{x^{2}}\right\}-\left\{\frac{A^{\prime}}{(t-1)^{2}}+\frac{B^{\prime}}{t(t-1)}+\frac{C^{\prime}}{t^{2}}\right\}
$$

thus obtaining $\Omega$ as a function of $t$ which, as will appear, vanishes identically when $A, B, C, A^{\prime}, B^{\prime}, C^{\prime}$ have the foregoing values.

I remark that this is, in effect, doing in a somewhat different form for the particular case what Goursat does for the general case, viz. starting from

$$
\Omega_{1}=\frac{x^{\prime \prime \prime}}{x^{\prime}}-\frac{3}{2}\left(\frac{x^{\prime \prime}}{x^{\prime}}\right)^{2}+x^{\prime 2}\left\{\frac{A}{(x-1)^{2}}+\frac{B}{x(x-1)}+\frac{C}{x^{2}}\right\}
$$

with values of $A, B, C$ which belong to the solution considered, he shows that this is a function of $t$ having no infinities other than $(0,1, \infty)$; that $\infty$ is not an infinity of the function or of the function multiplied into $t$, and that 0 and 1 are each of them a twofold infinity; that is, that the function is of the form

$$
\frac{L t^{2}+M t+N}{t^{2}(t-1)^{2}} \text { or } \frac{A^{\prime}}{(t-1)^{2}}+\frac{B^{\prime}}{t(t-1)}+\frac{C^{\prime}}{t^{2}} \text {. }
$$

Proceeding to carry out the process, we have

$$
\begin{gathered}
\frac{x^{\prime}}{x-1}=-\frac{1}{t-1}+\frac{3 Q^{\prime}}{Q}-\frac{2 R^{\prime}}{R} \\
\frac{x^{\prime}}{x}=\frac{2}{t}-\frac{1}{t-1}+\frac{3 P^{\prime}}{P}-\frac{2 R^{\prime}}{R}
\end{gathered}
$$

and from either of these equations, collecting and reducing,

$$
x^{\prime}=\frac{5 t P^{2} Q^{2}}{(t-1)^{2} R^{3}}
$$

where observe that, from the values of $x$ and $x-1$ respectively, it appears $\dot{a}$ priori that $t P^{2}$ and $Q^{2}$ must be factors in the numerator of $x^{\prime}$. From this value of $x^{\prime}$, we have

$$
\frac{x^{\prime \prime}}{x^{\prime}}=\frac{1}{t}-\frac{2}{t-1}+\frac{2 P^{\prime}}{P}+\frac{2 Q^{\prime}}{Q}-\frac{3 R^{\prime}}{R}
$$

and hence, $P^{\prime}$ and $Q^{\prime}$ being mere constants,

$$
\frac{x^{\prime \prime \prime}}{x^{\prime}}-\left(\frac{x^{\prime \prime}}{x^{\prime}}\right)^{2}=-\frac{1}{t^{2}}+\frac{2}{(t-1)^{2}}-\frac{3 R^{\prime \prime}}{R}-2\left(\frac{P^{\prime}}{P}\right)^{2}-2\left(\frac{Q^{\prime}}{Q}\right)^{2}+3\left(\frac{R^{\prime}}{R}\right)^{2}
$$


and consequently

$$
\begin{aligned}
\Omega= & -\frac{1}{t^{2}}+\frac{2}{(t-1)^{2}}-\frac{3 R^{\prime \prime}}{R}+2\left(\frac{P^{\prime}}{P}\right)^{2}-2\left(\frac{Q^{\prime}}{Q}\right)^{2}+3\left(\frac{R^{\prime}}{R}\right)^{2} \\
& -\frac{1}{2}\left(\frac{1}{t}-\frac{2}{t-1}+\frac{2 P^{\prime}}{P}+\frac{2 Q^{\prime}}{Q}-\frac{3 R^{\prime}}{R}\right)^{2} \\
& +A\left(-\frac{1}{t-1}+\frac{3 Q^{\prime}}{Q}-2 \frac{R^{\prime}}{R}\right)^{2} \\
& +B\left(-\frac{1}{t-1}+\frac{3 Q^{\prime}}{Q}-2 \frac{R^{\prime}}{R}\right)\left(\frac{2}{t}-\frac{1}{t-1}+\frac{3 P^{\prime}}{P}-\frac{2 R^{\prime}}{R}\right) \\
& +C\left(\frac{2}{t}-\frac{1}{t-1}+\frac{3 P^{\prime}}{P}-\frac{2 R^{\prime}}{R}\right)^{2} \\
& -\frac{C^{\prime}}{(t-1)^{2}}-\frac{B^{\prime}}{t(t-1)}-\frac{A^{\prime}}{t^{2}} .
\end{aligned}
$$

Putting for shortness

this equation gives

$$
\begin{aligned}
\frac{1}{t}=\alpha, & \frac{1}{t-1}=\beta, \frac{P^{\prime}}{P}=p, \frac{Q^{\prime}}{Q}=q, \frac{R^{\prime}}{R}=r \\
\Omega= & -\alpha^{2}+2 \beta^{2}-\frac{3 R^{\prime \prime}}{R}-2 p^{2}-2 q^{2}+3 r^{2} \\
& -\frac{1}{2}(\alpha-2 \beta+2 p+2 q-3 r)^{2} \\
& +A(-\beta+3 q-2 r)^{2} \\
& +B(-\beta+3 q-2 r)(2 \alpha-\beta+3 p-2 r) \\
& +C(2 \alpha-\beta+3 p-2 r)^{2} \\
& -C^{\prime} \alpha^{2}-B^{\prime} \alpha \beta-A^{\prime} \beta^{2},
\end{aligned}
$$

which is

$$
\begin{aligned}
& =\alpha^{2}\left(-\frac{3}{2}+4 C-C^{\prime}\right)-\frac{3 R^{\prime \prime}}{R}: \text { say it is }=L \alpha^{2}-\frac{48}{R} \\
& +\alpha \beta\left(2-2 B-4 C-B^{\prime}\right)+M \alpha \beta \\
& +\beta^{2}\left(A+B+C-A^{\prime}\right) \quad+N \beta^{2} \\
& +\alpha p(-2+12 C)+F a p \\
& +\alpha q(-2+6 B)+G \alpha q \\
& +\alpha r(3-4 B-8 C)+H a r \\
& +\beta p(4-3 B-6 C) \quad+F^{\prime} \beta p \\
& +\beta q(4-6 A-3 B) \quad+G^{\prime} \beta q \\
& +\beta r(-6+4 A+4 B+4 C) \quad+H^{\prime} \beta r \\
& +p^{2}(-4+9 C)+A^{\prime \prime} p^{2} \\
& +q^{2}(-4+9 A)+B^{\prime \prime} q^{2} \\
& +r^{2}\left(-\frac{3}{2}+4 A+4 B+4 C\right) \quad+C^{\prime \prime} r^{2} \\
& +q r(6-12 A-6 B)+F^{\prime \prime} q r \\
& +r p(6-6 B-12 C)+G^{\prime \prime} r p \\
& +p q(-4+9 B) \quad+H^{\prime \prime} p q \text {. }
\end{aligned}
$$


By decomposing $\alpha \beta$, $\alpha p$, \&c., into simple fractions, this becomes

$$
\begin{aligned}
\Omega= & L \alpha^{2}-\frac{48}{R} \\
& +M(-\alpha+\beta) \\
& +N \beta^{2} \\
& +F\left(-\frac{4}{5} \alpha+\frac{4}{5} p\right) \\
& +G\left(-\frac{5}{4} \alpha+\frac{5}{4} q\right) \\
& +H\left(-\frac{11}{8} \alpha+\frac{11 t+\frac{7}{8}}{R}\right) \\
& +F^{\prime \prime}(-4 \beta+4 p) \\
& +G^{\prime}(5 \beta-5 q) \\
& +H^{\prime}\left(\quad \beta+\frac{-8 t+19}{R}\right) \\
& +A^{\prime \prime} p^{2} \\
& +B^{\prime \prime} q^{2} \\
& +C^{\prime \prime} r^{2} \\
& +F^{\prime \prime} \frac{5}{12}\left(q-\frac{8 t-43}{R}\right) \\
& +G^{\prime \prime} \frac{4}{3}\left(p-\frac{8 t-13}{R}\right) \\
& +H^{\prime \prime} \frac{20}{9}(p-q) .
\end{aligned}
$$

This is

$$
\begin{aligned}
& \alpha^{2} L \\
& +\alpha\left(-M-\frac{4}{5} F-\frac{5}{4} G-\frac{11}{8} H\right) \\
& +\beta^{2} N \\
& +\beta\left(M-4 F^{\prime}+5 G^{\prime}+H^{\prime}\right) \\
& +p^{2} A^{\prime \prime} \\
& +p\left(4 F^{\prime}+\frac{4}{3} G^{\prime \prime}+\frac{20}{9} H^{\prime \prime}+\frac{4}{5} F^{\prime}\right) \\
& +q^{2} B^{\prime \prime} \\
& +q\left(-5 G^{\prime}-\frac{5}{12} F^{\prime \prime}-\frac{20}{9} H^{\prime \prime}+\frac{5}{4} G\right) \\
& +r^{2} C^{\prime \prime} \\
& +\frac{1}{R}\left\{-48+H\left(11 t+\frac{7}{8}\right)+H^{\prime}(-8 t+19)-\frac{5}{12} F^{\prime \prime}(8 t-43)-\frac{4}{3} G^{\prime \prime}(8 t-13)\right\} .
\end{aligned}
$$

This should be identically $=0$; making $A^{\prime \prime}=0, B^{\prime \prime}=0, C^{\prime \prime}=0$, we find

$$
A=\frac{4}{9}, B=-\frac{37}{32}, C=\frac{4}{9} ;\left(A+B+C=\frac{3}{8}\right) .
$$


and thence

$$
\begin{gathered}
F=\frac{10}{3}, \quad G=-\frac{61}{12}, \quad H=\frac{3}{2} ; \quad F^{\prime}=\frac{23}{8}, \quad G^{\prime}=\frac{23}{8}, \quad H^{\prime}=-\frac{9}{2} \\
F^{\prime \prime}=\frac{15}{4}, \quad G^{\prime \prime}=\frac{15}{4}, \quad H^{\prime \prime}=-\frac{68}{9} .
\end{gathered}
$$

These values make the coefficients of $p$ and $q$ to be each $=0$; and they make the coefficient of $R$ to be identically $=0$, viz. we have

and

$$
0=-48+\frac{7}{8} H+19 H^{\prime}+\frac{215}{12} F^{\prime \prime}+\frac{52}{3} G^{\prime \prime},
$$

$$
0=11 H-8 H^{\prime}-\frac{10}{3} F^{\prime \prime}-\frac{32}{3} G^{\prime \prime}
$$

We have, moreover,

$$
L=\frac{5}{18}-C^{\prime}, \quad M=\frac{365}{144}-B^{\prime}, \quad N=\frac{3}{8}-A^{\prime} ;
$$

and the coefficients of $\alpha$ and $\beta$ are $=-M+\frac{13}{8}$ and $M-\frac{13}{8}$ respectively; hence the coefficients of $\alpha^{2}, \beta^{2}, \alpha$ and $\beta$ will all vanish if only $L=0, M=\frac{13}{8}, N=0$, that is,

$$
A^{\prime}=\frac{3}{8}, \quad B^{\prime}=\frac{131}{144}, \quad C^{\prime}=\frac{5}{8} ;
$$

and we have thus identically $\Omega=0$, if only $A, B, C, A^{\prime}, B^{\prime}, C^{\prime}$ have the abovementioned values.

C. XIII. 